\title{
Post-traumatic stress symptoms are associated with better performance on a delayed match-to-position task
}

\author{
Meghan D Caulfield ${ }^{\text {Corresp., }}{ }^{1,2}$, Catherine E Myers ${ }^{\text {Corresp. } 2,3}$ \\ 1 Department of Psychology, Lafayette College, Easton, Pennsylvania, United States of America \\ 2 Neurobehavioral Research Laboratory, VA New Jersey Health Care System, East Orange, New Jersey, United States of America \\ 3 Department of Pharmacology, Physiology \& Neuroscience, New Jersey Medical School, Newark, New Jersey, United States of America \\ Corresponding Authors: Meghan D Caulfield, Catherine E Myers \\ Email address: caulfiem@lafayette.edu, catherine.myers2@va.gov
}

Many individuals with posttraumatic stress disorder (PTSD) report experiencing frequent intrusive memories of the original traumatic event (e.g. flashbacks). These memories can be triggered by situations or stimuli that reflect aspects of the trauma and may reflect basic processes in learning and memory, such as generalization. It is possible that, through increased generalization, non-threatening stimuli that once evoked normal memories become associated with traumatic memories. Previous research has reported increased generalization in PTSD, but the role of visual discrimination processes has not been examined. To investigate visual discrimination in PTSD, 143 participants (Veterans and civilians) self-assessed for symptom severity were grouped according to the presence of severe PTSD symptoms (PTSS) vs. few/no symptoms (noPTSS). Participants were given a visual match-to-sample pattern separation task that varied trials by spatial separation (Low, Medium, High) and temporal delays (5, 10, 20, 30 seconds). Unexpectedly, the PTSS group demonstrated better discrimination performance than the noPTSS group at the most difficult spatial trials (Low spatial separation). Further assessment of accuracy and reaction time using diffusion drift modeling indicated that the better performance by the PTSS group on the hardest trials was not explained by slower reaction times, but rather a faster accumulation of evidence during decision making in conjunction with a reduced threshold, indicating a tendency in the PTSS group to decide quickly rather than waiting for additional evidence to support the decision. This result supports the need for future studies examining the precise role of discrimination and generalization in PTSD, and how these cognitive processes might contribute to expression and maintenance of PTSD symptoms. 
1 Post-traumatic stress symptoms are associated with better performance on a delayed match-to-

2

3

4

5

6

7

8

9

10

11 Corresponding Author:

12 Catherine E. Myers, Ph.D.

13 Catherine.myers2@va.gov position task

$$
\text { Meghan D. Caulfield }{ }^{1,2} \text {, Catherine E. Myers }{ }^{2,3}
$$

1. Department of Psychology, Lafayette College, Easton PA USA

2. Neurobehavioral Research Laboratory, VA New Jersey Health Care System, East Orange, NJ USA

3. Department of Pharmacology, Physiology \& Neuroscience, New Jersey Medical School, Rutgers University, Newark NJ USA 
Many individuals with posttraumatic stress disorder (PTSD) report experiencing frequent intrusive memories of the original traumatic event (e.g. flashbacks). These memories can be triggered by situations or stimuli that reflect aspects of the trauma and may reflect basic processes in learning and memory, such as generalization. It is possible that, through increased generalization, non-threatening stimuli that once evoked normal memories become associated with traumatic memories. Previous research has reported increased generalization in PTSD, but the role of visual discrimination processes has not been examined. To investigate visual discrimination in PTSD, 143 participants (Veterans and civilians) self-assessed for symptom severity were grouped according to the presence of severe PTSD symptoms (PTSS) vs. few/no symptoms (noPTSS). Participants were given a visual match-to-sample pattern separation task that varied trials by spatial separation (Low, Medium, High) and temporal delays (5, 10, 20, 30 seconds). Unexpectedly, the PTSS group demonstrated better discrimination performance than the noPTSS group at the most difficult spatial trials (Low spatial separation). Further assessment of accuracy and reaction time using diffusion drift modeling indicated that the better performance by the PTSS group on the hardest trials was not explained by slower reaction times, but rather a faster accumulation of evidence during decision making in conjunction with a reduced threshold, indicating a tendency in the PTSS group to decide quickly rather than waiting for additional evidence to support the decision. This result supports the need for future studies examining the precise role of discrimination and generalization in PTSD, and how these cognitive processes might contribute to expression and maintenance of PTSD symptoms. 
39

40

41

42

43

44

45

46

47

48

49

50

51

52

53

54

55

56

57

58

59

60

61

62

63

64

65

66

67

68

69

70

71

72

73

74

75

76

77

78

79

80

81

When exposed to a traumatic event, some individuals develop posttraumatic stress disorder (PTSD), including re-experiencing symptoms such as frequent intrusive memories (e.g. flashbacks), believed to be triggered by situations that resemble aspects of the traumatic event (e.g. Elzinga \& Bremner, 2002; Brewin \& Holmes, 2003; Brewin, Gregory \& Lipton, 2010). Learning and memory processes such as generalization and discrimination may underlie reexperiencing symptoms in PTSD. For example, patients with PTSD may be impaired at discrimination, the ability to distinguish and respond differently to similar stimuli, and/or may show increased generalization, the ability to take what is learned about one stimulus and apply it to other. A consequence of increased generalization is that stimuli, which once evoked normal memories, become capable of evoking traumatic memories (e.g. Tryon, 1998; van Meurs et al., 2014).

Evidence that PTSD is associated with overgeneralization emerges from differential conditioning studies, in which patients with PTSD often show reduced discrimination between fear and safety cues (Orr et al., 2000; Peri, Ben-Shakhar \& Orr, 2000; Blechert et al., 2007; Jovanovic, Norrholm \& Blanding, 2010). Poor differentiation of fear and safety cues is conceptualized as overgeneralization of fear learning, such that similar fear responses are evoked by both cues. Recent research provides a behavioral link between discrimination and generalization, demonstrating that behavioral pattern separation is related to increased fear generalization in healthy young adults (Lange et al, 2017). Given that patients with PTSD demonstrate reduced discrimination learning of fear and safety signals, it is possible that impaired discrimination or generalization may extend to more neutral learning tasks, such as computer-based associative learning tasks. In such tasks, participants are first challenged to learn a series of stimulusresponse mappings by trial and error, such as picking the rewarded object from each pair of objects, or learning which of two stimuli should be paired with a target. Later, task demands change by altering irrelevant features of familiar stimuli or by presenting familiar objects in new pairings, making it possible to assess how well participants generalize previously learned associations to the new stimuli or pairings. A body of studies now support the idea that the hippocampal region - although not strictly required for the initial stimulus-response learning contributes during learning by setting up stimulus representations to support subsequent generalization. Thus, amnesic patients with hippocampal-region damage can learn the initial associations, but then perform at or near chance on the generalization phase (Myers et al., 2008a), as do nondemented elderly with hippocampal atrophy consistent with prodromal Alzheimer's disease (Myers et al., 2002; 2003; 2008b). Further, functional imaging studies in healthy adults have shown associations between hippocampal region activity at learning, and successful generalization (Myers et al., 2008b; Shohamy \& Wagner, 2008; Fera et al., 2014).

The hippocampus is also implicated in PTSD. Structural neuroimaging studies show reduced hippocampal volume in patients with PTSD (Gurvits et al., 1996; Villarreal et al., 2002; Gilbertson et al., 2002a), as well as a relationship between overgeneralization of negative context and bilateral hippocampal volume in PTSD (Levy-Gigi et al., 2015). Functional neuroimaging studies suggest reduced or abnormal hippocampal activity in PTSD (Astur et al., 2006; Werner et al., 2009; Milad et al., 2009). Given the reduced hippocampal volume and abnormal hippocampal activity observed in PTSD, it might be expected that Veterans with PTSD would perform similar to patients with medial temporal (hippocampal) amnesia and nondemented 
82 elderly with hippocampal region damage: i.e., demonstrating spared learning but reduced generalization on these associative learning tasks. However, several prior studies have now reported the opposite pattern of increased generalization by Veterans with clinically-diagnosed PTSD or severe current PTSD symptoms, compared to peers with few/no PTSD symptoms (Kostek et al., 2014; Anastasides et al., 2015; Levy-Gigi et al., 2015) suggesting a more complicated picture of the hippocampus in PTSD.

One explanation is that rather than generalizing, participants with PTSD symptoms may simply be less able to distinguish or discriminate subtle differences between visual stimuli. Poor visual discrimination could promote increased generalization simply because participants fail to detect small differences between visual stimuli. Studies examining visual discrimination in humans frequently use briefly presented images that are very similar (but not identical) to previously presented objects, requiring a high degree of visual discrimination that may reflect multiple underlying processes, such as decreased attention or motivation to learn during the acquisition phase. Discrimination difficulties in such tasks may also reflect a decrease in pattern separation function, a process of comparing new inputs to prior inputs that has consistently been shown to depend on the dentate gyrus of the hippocampus (Bakker et al., 2008; Lacy et al., 2011; Reagh \& Yassa, 2014; Berron et al., 2016).

To investigate whether decreased discrimination occurs in a visual discrimination task we conducted Experiment 1, which used a sample of Veterans from the same population as several of the earlier generalization studies, along with an additional civilian group. All participants were self-assessed for PTSD symptom severity, as in earlier studies, and grouped according to presence or absence of severe PTSD symptoms (PTSS). Participants were then administered a spatial discrimination task in the form of a visual match-to-sample test, where subjects first saw a target object at a particular screen location, and then after a short temporal delay were challenged to distinguish the target from an identical object presented at a new location. If the PTSS group performed worse on the task, this would suggest that the pattern of increased generalization observed in prior studies might be explained either as a reduction of visual discrimination, or inability to maintain attention and concentration. If no such deficit were found, this would suggest that the increased generalization observed in prior studies is more likely to be due to active processes, such as imbalance in hippocampal or other brain substrates normally mediating the tradeoff between generalization and specificity in learning.

Following Experiment 1, which documented between-group differences, Experiment 2 was conducted to use mathematical analysis techniques in an attempt to better understand possible latent cognitive processes that could differ between groups to produce the observed behavioral pattern.

\section{EXPERIMENT 1: SPATIAL DISCRIMINATION TASK}

To assess spatial pattern separation, a visual delayed match-to-sample task was modified from Holden et al. (2012), which in turn adapted the task for humans based on rat spatial and temporal discrimination paradigms (Gilbert, Kesner \& Lee, 2001; Gilbert \& Kesner, 2002). Each trial included a sample phase, in which participants viewed a single circle on the computer screen, followed by a choice phase in which participants identified which of two circles was in the previously-viewed location. Choice phase stimuli were separated by one of four possible spatial separations: $0 \mathrm{~cm}, 0.5 \mathrm{~cm}, 1.0 \mathrm{~cm}$, and $1.5 \mathrm{~cm}$. Smaller spatial separations between the sample 
125

126

127

128

129

130

131

132

133

134

135

136

137

138

139

140

141

\section{2}

143

144

145

146

147

148

149

150

151

152

153

154

155

156

157

158

159

160

161

162

163

164

165

phase and choice phase are more difficult, requiring greater pattern separation processes, and presumably greater reliance on the dentate gyrus of the hippocampus. In their study, Holden and colleagues (2012) used this procedure to examine pattern separation processes in cognitively normal young adults and older adults. As expected, when spatial separation increased (and the task became easier) performance improved in both groups. However, older adults did not demonstrate the same degree of benefit of increased spatial separation as younger adults, suggesting less efficient pattern separation processes in older adults and implicating age-related changes in the dentate gyrus of the hippocampus. Holden and colleagues further demonstrated hippocampal contributions in their spatial discrimination task by using a standardized test of verbal memory to compare cognitively unimpaired to cognitively impaired older adult subgroups. This comparison revealed that the cognitively impaired group had significantly poorer performance compared to the unimpaired group, again with the largest group differences at the largest spatial separation $(1.5 \mathrm{~cm})$. This result highlights that the task is sensitive to individual differences in spatial discrimination in aging and implicates the hippocampus as playing a possible role in mediating visual spatial separation tasks. The present study extended the use of this task to examine individual differences in spatial discrimination in PTSD.

\section{METHODS}

\section{Participants}

143 participants, including 90 Veterans and 57 civilians (never served in the military), were recruited from the East Orange VA Medical Center and surrounding community, by flyer and word-of-mouth referral. Participants were paid \$20/hour for participation in a single 2-hour session. All participants signed written statements of informed consent at the start of the session. Study procedures were approved by the Institutional Review Board at VA NJHCS (Approval \#: 01161) and conformed to guidelines established by the Declaration of Helsinki and the Federal Government for the protection of human subjects.

Because the Veteran sample included an overrepresentation of males (about $85 \%$ male), we intentionally oversampled females in the civilian sample to adequately power analyses of the effects of gender, as well as PTSS and military service. Additionally, the Veteran group was subdivided post-hoc into combat-exposed vs. non-combat-exposed groups (see below for procedure), resulting in three participant groups: civilian, non-combat Veteran, and combat Veteran.

One Veteran participant withdrew from the study before completing all the tasks; this Veteran's data were excluded from subsequent analysis.

The resulting set of 142 participants ( 87 Veterans and 55 civilians) included 86 males and 56 females, with a mean age of 51.1 years $(S D$ 13.5) and mean education level of 15.0 years $(S D$ 2.7). Asked to self-report race, 96 participants self-identified as Black, African, or AfricanAmerican; 23 as White or Caucasian, 11 as Mixed-Race, and 11 as Other (including 1 Native American); 1 subject declined to specify. Asked about ethnicity, 6 participants self-identified as Hispanic, 134 as non-Hispanic, and 2 declined to specify.

Asked about specific conflicts in which they had served, 32 Veterans specified Vietnam, 14 specified Gulf War/Operation Desert Storm, 12 specified Operation Enduring 
166

Freedom/Operation Iraqi Freedom, 8 specified other conflicts (e.g. Bosnia, Lebanon, Somalia), and 29 reported no specific conflict or peacetime service. Numbers sum to greater than 87 due to some Veterans whose service spanned multiple conflicts.

\section{Procedures}

Testing took place in a quiet testing room, with the participant seated at a table for paper-andpencil questionnaires and tasks, or at a comfortable viewing distance from a Macintosh i-mac computer (22" screen) for computer-based tasks. All participants received a battery of questionnaires and tasks, as detailed below. As permitted within the constraints of the 2-hour testing session, some participants also received additional tasks (e.g. piloting of new behavioral tasks or questionnaires); those data are not reported here.

\section{Questionnaires}

All participants received the Posttraumatic Stress Checklist (PCL-C; (Blanchard et al., 1996), a 17-question self-report measure of the presence and frequency of PTSD symptoms; symptoms are rated according to how much they have "bothered" the participant in the past month, from not at all (1) to extremely (5). The PCL-C is highly predictive of clinician-rated PTSD in Veterans (Weathers et al., 1993; Blanchard et al., 1996; Lunney et al., 2014). Specific PCL-C questions correspond to $D S M-I V$ symptom clusters including re-experiencing, avoidance/numbing, and increased arousal. PCL scores of 50+ are predictive of PTSD in military samples (Weathers et al., 1993; Blanchard et al., 1996). Accordingly, as in prior studies, (Radell, et al., 2017) we used this cutoff to separate participants according to presence or absence of current, severe PTSD symptoms (PTSS).

Veteran participants also received the Combat Exposure Scale (CES), which assesses exposure to stressful military events (Keane, Fairbank \& Caddell, 1989). Following prior studies (Ginsberg et al., 2008; Myers, VanMeenen \& Servatius, 2012; Radell et al., 2017), Veterans scoring 8 or higher on the CES were classified as having a history of exposure to combat, and those scoring below 8 were exposed as not having combat exposure.

\section{Trail-Making Test (TMT)}

In order to rule out the possibility that any group differences in our spatial discrimination task might reflect differences in motor or cognitive flexibility, all participants completed the trailmaking test (TMT), a standard neuropsychological tool to assess visual attention, sequencing, mental flexibility and motor function (Spreen \& Strauss, 1998). In Part A, participants are presented with a sheet of paper containing 25 encircled numbers, placed in various locations around the page, and use a pencil to "connect the dots" in numerical order as quickly as possible. Part B is similar, except that the page contains 25 encircled numbers and letters, and participants connect the dots alternating letters and numbers (i.e., 1-A-2-B-3-C...) as quickly as possible. If the subject makes an error, the experimenter calls attention immediately and has the participant proceed from the point where the mistake occurred. Performance is scored as time in seconds to complete each part, with errors counting only in increased time of performance. 
Spatial Discrimination Task

207 The spatial discrimination task is a computer-based test of spatial and temporal discrimination

208 adapted from Holden and colleagues (2012) and presented on a Macintosh computer with 9x12"

209 screen programmed in the SuperCard development environment (Solutions Etcetera, Pollock

210 Pines CA). The task involves the presentation of a fixation cross $(3 \mathrm{sec})$, followed by a target dot

211 (a small filled circle about $50 \mathrm{~mm}$ in diameter) which appears at the target location which is

212 always located at the horizontal midline of the screen, offset by a random amount $0 \pm 3 \mathrm{~cm}$ to the

213 left or right of vertical midline. Following a variable delay, the participant is presented with a

214 second fixation cross and then presented with two dots (the original target dot and a second

215 distractor dot). The participant is asked to identify which dot was the original target by making a

216 key press of "LEFT" or "RIGHT" on a masked keyboard (Figure 1). Participants can use either

217 or both hands to enter responses but cannot touch the screen or otherwise physically "hold" the target location during the delay period. The experiment consists of three practice phases: one phase to familiarize the participants with the dots task ( $3.5 \mathrm{~cm}$ spatial separation, 2 trials $)$, a second phase asking participants to read randomly presented numerals ( 0 to $9 ; 1.25 \mathrm{~cm}$ tall) aloud, and a third phase combining the two so that participants perform the numeral naming task in the delay between target and choice ( 2 trials). The testing phase is similar in format to the third practice phase, and includes two blocks of 12 trials, including one trial with each of several spatial separations (Low $=0.75 \mathrm{~cm}$, Medium $=1.75 \mathrm{~cm}, \mathrm{High}=2.75 \mathrm{~cm}$ ) at each of several delays $(5,10,20,30$ seconds). Trial order is randomized within a block, and placement of the distractor location to the left or right of the target is randomized across trials. After every sixth trial, a "rest" screen, appears, offering the subject a chance to take a short break to rest his/her eyes for a few moments, then click the mouse button to resume trials. The entire task took about 15-20 minutes to complete.

\section{$\underline{\text { Data Analysis }}$}

Statistical analyses were conducted using SPSS version 22 statistical software.

One-way ANOVAs were used to compare the three groups in age and education level, with posthoc independent-samples t-tests to further explore significant effects. Chi-square test for independence was used to compare male/female distribution and rate of PTSS cases between groups. Based on the results (summarized below and in Table 1), we opted to include gender in all our analyses as a between-subjects factor as there have been observations of sex differences in prevalence of PTSD (McLean et al., 2011) as well as learning differences indicating females show enhanced discrimination and generalization during learning (Day et al., 2017). Since there were no observed differences between groups in education level, this variable was not used as a covariate in subsequent analyses. However, since age is known to affect various speededresponse tasks including the TMT (e.g. Spreen \& Strauss, 1998), we included age as a covariate in analyses of TMT and the spatial discrimination task (after verifying assumption of homogeneity of variance across groups was satisfied). complete each phase (Parts A and B), between-subjects factors of group (civilian, non-combat 
246

Veteran, combat Veteran), gender (male, female), and PTSS (noPTSS, PTSS), and covariate of age.

For the spatial discrimination test, percent correct (averaged across two trials) was scored with each combination of spatial and temporal separation. Mixed ANOVA was then conducted with within-subjects factors of spatial separation (3 levels) and temporal separation (4 levels), between-subjects factors of group, gender, and PTSS, and covariate of age.

RT data were also collected for each combination of spatial and temporal separation (averaged across two trials at each combination). The computer program recorded RT in Mac OS ticks $\left(1 / 60^{\text {th }} \mathrm{s}\right)$, which were converted to ms prior to analysis. Because RT data can be vulnerable to outliers (Ratcliff, 1993), data cleansing was performed and any trials with RT $<250 \mathrm{msec}$ or $>$ $10 \mathrm{~s}$ were dropped from analysis. First, very fast RTs (e.g. $<250 \mathrm{~ms})$ can indicate that the subject has (intentionally or unintentionally) pressed the key before onset of stimulus presentation; accordingly, trials with such "fast-RT" responses were excluded from RT analysis. Second, especially long RTs are often considered to be the result of other, interfering processes (such as attentional lapse). In healthy young adults given a speeded task, this criterion is often taken to be about 3-5 s. However, in older subjects, psychiatric populations, or individuals on various psychoactive drugs, mean responses are much slower, and the criterion is often extended, to avoid dropping large numbers of trials from analysis (e.g. Ratcliff, 1993; Herzallah et al., 2017). In the current study, trials with RTs longer than 10s were excluded, which allowed retention of a large majority of trials while excluding a small number that lay outside the usual range.

After data cleansing, mean RT was computed for correct and incorrect responses at each spatial and temporal separation, and across the task as a whole, for subjects who had at least one trial of the relevant type remaining after data cleansing. Mixed ANOVA was then conducted with within-subjects factors of response (correct vs. incorrect) and spatial separation, betweensubjects factors of group, gender, and PTSS, and covariate of age.

Threshold for significance was set at alpha $=.05$. Where omnibus tests were significant and multiple post-hoc tests were conducted to explore significant results, Bonferonni correction was used to adjust significance thresholds to protect against Type I error; adjusted threshold is only reported where p-values fall below .05 but above the corrected threshold. ANOVA was computed based on Type III SS calculations. Where data failed statistical assumptions for parametric tests (e.g. assumptions of sphericity and equal variance) appropriate corrections were used to adjust degrees of freedom (e.g. Greenhouse-Geisser for ANOVA, Welch's $t$ for $t$-test).

\section{RESULTS}

\section{Questionnaire Results}

Based on CES scores, 33 of the 87 Veteran participants were classified as having a history of exposure to combat, and the remaining 54 were classified as non-combat Veterans. Together with the civilians $(n=55)$, this resulted in three participant groups, as summarized in Table 1. The groups differed significantly in age $\left(F(2,139)=4.80, p=.010\right.$, partial $\left.\eta^{2}=0.065\right)$; post-hoc pairwise $t$-tests confirmed that the non-combat Veteran group was significantly older than the civilian group $(t(107)=3.11, p=.002)$ but the combat Veteran group did not differ from either the non-combat Veteran group (Welch's $t(51.65)=1.93, p=.059)$ or the civilian group $(t(86)=$ 
287

288

289

290

291

292

293

294

295

296

297

298

299

300

301

302

303

304

305

306

307

308

309

310

311

312

313

314

315

316

317

318

319

320

321

322

323

324

325

326

$0.70, p=.488)$. Given the group differences in age, age was included as a covariate in subsequent analyses. There were no group differences in education $(F(2,138)=1.87, p=.158)$. As expected, the difference in gender distribution was significant $\left(\chi^{2}(2, N=142)=43.58, p<\right.$ .001 , Cramer's V $=0.554$ ); specifically, there were proportionally more females in the civilian group than in the non-combat Veteran group(Yates-corrected $\chi^{2}(1, N=109)=23.91, p<.001$, Cramer's V $=0.487$ ) or combat Veteran group (Yates-corrected $\chi^{2}(1, N=88)=30.93, p<.001$, Cramer's $\mathrm{V}=0.616$ ) groups, although the two Veteran groups did not differ (Yates-corrected $\chi^{2}(1, N=87)=2.15, p=0.143$,

Table 1 also shows mean PCL scores for each group. ANOVA (with factors of group and gender and covariate of age) confirmed a significant effect of group $(F(2,135)=15.45, p<.001$, partial $\left.\eta^{2}=0.186\right)$ with no main effect of gender $(F(1,135)=0.10, p=.757)$ and no gender-group interaction $(F(2,135)=1.79, p=.170)$, although the effect of the covariate was significant $(F(1,135)=7.39, p=.007)$. Post-hoc pairwise $t$-tests revealed that the civilian group had lower PCL scores than either the combat Veteran $(t(86)=5.38, p<.001)$ or non-combat Veteran group $(t(107)=5.24, p<.001)$; the two Veteran groups did not differ $(t(85)=0.96, p=.339)$. In Veterans, CES did not correlate with PCL scores, $r=.128, n=87, p=.238$.

Using the cutoff of PCL-C $\geq 50$ to define PTSS, 71 participants (50.0\%) met criteria for PTSS across the three groups (civilian, non-combat, combat). The distribution of PTSS cases across groups differed significantly $\left(\chi^{2}(2, N=142)=26.72, p<.001\right.$, Cramer's V=0.434), with proportionately fewer cases among civilians than Veteran groups (both Yates-corrected $\chi^{2}>$ 14.0, both $p<.001$ ); however, rates did not differ between the two Veteran groups (Yatescorrected $\chi^{2}=1.37, p=.241$ ).

\section{Trail-Making Test}

Unsurprisingly, scores on TMT parts A and B were highly correlated (Pearson's $r=.617, p<$ .001 ), with subjects who scored high (poor performance) on part A tending to also score high (poor performance) on part B. However, TMT part A and B scores did not correlate with either CES or PCL (all $p$ 's > .05). Mixed measures ANOVA with repeated measures factor of TMT part A and part B score with between-subjects factors of group, gender and PTSS status, revealed a significant effect of part $(F(1,130)=123.57, p<.001)$, with subjects generally taking longer to complete part B $(M=102.7 \mathrm{sec}, S D=48.0)$ than part A $(M=46.9 \mathrm{sec}, S D=20.6)$, but with no effects of gender, group, or PTSS status and no interactions (all $p$ 's $>.070$ ).

\section{Spatial Discrimination Task: Percent Correct Responding}

Consistent with previous studies reporting poorer spatial discrimination in aging, our sample also showed a negative correlation between task performance (total percent correct) and age, with younger subjects outperforming older (Pearson's $r=-0.227, p=.007, r^{2}=0.052$ ). The correlation remained after partialing out effects of group $\left(r=-0.264, p=.002, r^{2}=0.070\right)$. Accordingly, age was entered as a covariate in the subsequent analyses of task performance.

Figure 2 shows mean percent correct across the four delays (Figure 2A) and the three spatial separations (Figure 2B) for PTSS collapsed across civilian and Veteran groups. As expected, for the noPTSS group, performance was better at high spatial separation than low spatial separation, 
327

328

329

330

331

332

333

334

335

336

337

338

339

340

341

342

343

344

345

346

347

348

349

350

351

352

353

354

355

356

357

358

359

360

361

362

363

364

365

366

367

and better for shorter delay than for longer delay. However, the PTSS group appeared less sensitive to reductions in spatial separation or increasing temporal delay.

Mixed ANOVA with Greenhouse-Geisser correction was used to examine performance on the spatial discrimination task at varying temporal and spatial separations. There was an interaction between PTSS and spatial separation $\left(F(1.85,238.01)=3.35, p=.040\right.$, partial $\left.\eta^{2}=0.025\right)$, as well as a significant within-subjects effect of spatial separation (i.e., worse performance as separation decreased, $F(1.85,238.00)=5.52, p=.005$, partial $\left.\eta^{2}=0.0741\right)$. There was no within group effect observed for temporal separation (i.e., no effect of delay between target and choice, $p=$ .430 ). No other interactions or effects reached significance (all $p$ 's $>.050$ ), except for the effect of the covariate age $(F(1,129)=5.49, p=.021)$.

To further investigate the interaction between spatial separation and PTSS, data were collapsed over the four temporal separations, as shown in Figure 2B, and Bonferroni-corrected paired $t$ tests run at each spatial separation. Participants meeting PTSS criteria outperformed noPTSS participants specifically at the low (i.e. most difficult) spatial separation $(t(140)=.003$, Cohen's $d=0.485)$, but not at the medium $(t(140)=0.90, p=.370, \mathrm{~d}=0.169)$ or high (i.e. easiest) spatial separation $(t(140)=0.13, p=.898, d=0.022)$.

The above analyses included all 142 subjects, including some who performed at or near chance in terms of percent correct calculated across all 24 trials. To determine if those who "solved" the task were driving observed differences in the above analyses. We also analyzed the data from only those participants who made at least 17 out of 24 correct responses $(70.8 \%$ correct), i.e. significantly better than chance based on binomial calculations, $\mathrm{P}(\mathrm{X}>16)=.032)$. This included 34 of 71 PTSS participants and 22 of 71 noPTSS participants, a difference that fell short of statistical significance (Yates-corrected $\mathrm{c} 2(1)=3.57, p=.059$ ). When performance was analyzed in this subset, results look qualitatively similar to those shown for the full dataset in Figure 2; specifically, the PTSS group continued to outperform the noPTSS group at the low but not medium or high spatial separation (Bonferroni-corrected paired $t$-tests, Low separation: $t(54)$ $=2.90, p=.005, \mathrm{~d}=0.716$; Medium separation: $t(54)=0.10, p=.922, d=0.042$; High separation: $t(54)=2.06, p=.044, d=0.563)$.

Finally, performance at the lowest spatial separation was significantly correlated with PCL scores $\left(r=.229, n=142, p=.006, \mathrm{r}^{2}=0.052\right)$, although performance at the higher spatial separations was not (Medium: $r=.130, p=.122, \mathrm{r}^{2}=0.169$; High: $r=.064, p=.451, \mathrm{r}^{2}=0.004$ ). The correlation between PCL scores and performance at the lowest spatial separation held even when considering only the $n=56$ "solvers" $\left(r=.396, p=.003, \mathrm{r}^{2}=0.157\right)$. There was no correlation between performance at any spatial separation and CES (all $p$ 's > .08).

\section{Spatial Discrimination Task: Reaction Time}

During data cleansing for RT analysis, a total of 75 trials $(2.2 \%$ of total $)$ were excluded as longRT $(\mathrm{RT}>10 \mathrm{~s})$; the vast majority of participants (129 of 142) made 1 or fewer long-RT responses, and only three subjects (including one who made 12 long-RT responses) made more than 5 long-RT responses. Additionally, 8 "fast-RT" trials $(\mathrm{RT}<250 \mathrm{~ms})$ were excluded: one subject made 5 responses with $\mathrm{RT}=0$ and two subjects made 1-2 responses with $\mathrm{RT}=0$. No other $\mathrm{RT}<250 \mathrm{~ms}$ were recorded. 
368

369

370

371

372

373

374

375

376

377

378

379

380

381

382

383

384

385

386

387

388

389

390

391

392

393

394

395

396

397

398

399

400

401

402

403

404

405

406

407

408
Mean RT data for trials at each spatial separation are shown in Figure 3A, for trials with correct and incorrect responses. However, many subjects did not have RT scores for all combinations of temporal and spatial separation; in particular, 32 subjects did not make any incorrect responses on any trials at the largest spatial separation). For those 101 subjects where all six mean RT scores were available, mixed ANOVA on mean RT for correct and incorrect responses on trials at each spatial separation showed a within-subjects effect of response, as subjects responded faster on correct than incorrect trials $\left(F(1,88)=11.868, p=.001\right.$, partial $\eta^{2}=0.119$; there were also significant three-way interactions between trial type, group, and PTSS $(F(2,88)=3.46, p=$ .036 , partial $\left.\eta^{2}=0.073\right)$ and between trial type, PTSS and gender $(F(1,88)=4.45, p=.037$, partial $\eta^{2}=0.049$ ); no other between-subjects effects of group, gender, or PTSS, and no interactions, approached significance (all $p$ 's $>.100$ ) except the interaction between trial type and the covariate age $(F(1,88)=6.84, p=0.010)$. Post-hoc testing to explore the three-way interactions (i.e., mixed ANOVA to explore type-gender and type-group interactions in the PTSS and noPTSS subgroups) did not reveal significant effects (all $p>.050)$.

Because the above analysis excluded a number of subjects, a secondary analysis was conducted on the RT scores, collapsing across all spatial separations; allowing mean RT for correct and incorrect trials to be computed for all except a single subject in the PTSS group who made no incorrect responses on the task (Figure 3B). There was again a within-subjects effect of response type $F(1,129)=4.11, p=.045$, partial $\left.\eta^{2}=0.031\right)$; no other effects or interactions approached significance all $p$ 's $>.100)$.

In summary, although the RT data showed an expected within-subjects effect of faster responding on correct than incorrect trials, neither analysis showed evidence of a PTSS (or gender) effect on RTs. Because the direction of effect - with the PTSS group showing better performance at the most difficult spatial separation, without evidence of slower RT mediating this accuracy - was unexpected, we turned in Experiment 2 to explore latent cognitive processes that could underlie these results.

\section{EXPERIMENT 2}

Two-choice forced-response tasks, such as the spatial discrimination task studied in Experiment 1, generally involve a speed-accuracy tradeoff, whereby better performance can be achieved at the cost of slower RT, or faster RT at the expense of reduced accuracy. Because these two behavioral variables interact, analysis of either alone does not give a complete picture of the underlying cognitive processes mediating performance.

To address this issue, various mathematical models have been developed. Drift diffusion models (DDMs) represent a class of model commonly used to infer latent cognitive processes underlying two-choice decision-making, and to link these processes to neural mechanisms (Ratcliff, 1978; Ratcliff \& McKoon, 2008; Ratcliff et al., 2016).

DDMs assume that organisms make rapid (often implicit) decisions based on accumulation of noisy information over time. In the case of a two-choice forced response task, as schematized in Figure 4, the decision process on each trial begins at a starting point " $z$ ". Noisy accumulation of information pushes the decision process towards one of two boundaries representing criteria for the two possible responses. When a boundary is reached, the corresponding response is executed. 
409

410

411

412

413

414

415

416

417

418

419

420

421

422

423

424

425

426

427

428

429

430

431

432

433

434

435

436

437

438

439

440

441

442

443

444

445

446

447

448

449

450

451

452

Within-trial variability (noise) means that the same information does not always produce the same RT or even the same behavioral responses.

Central to the DDM approach is the idea that different individuals can be described in terms of different "parameters" that together govern the decision process. These parameters include the rate of information processing (called the drift rate, v), the a priori response bias (i.e. starting point $\mathrm{z}$ may be closer to one boundary than the other), and nondecision time, Ter, which includes stimulus processing and response execution time. Subjects can also differ in the distance between boundaries (a), a parameter often assumed to encapsulate "response caution" since higher values mean that more evidence must be accumulated before a boundary is reached and a response selected. Later elaborations of the DDM have included additional variables encoding intertrial variability, although these parameters may increase model complexity without necessarily increasing explanatory power (Lerche \& Voss, 2016).

Several methods have been proposed for fitting the DDM to individual subject data, i.e. using a participant's observed RT and accuracy measurements to infer (or "recover") parameters such as $\mathrm{v}$, Ter, a, and $\mathrm{z}$ that best describe that participant's observed trial-by-trial behavior (Ratcliff \& Tuerlinckx, 2002; Ratcliff \& McKoon, 2008; Wiecki, Sofer \& Frank, 2013; Voss, Voss \& Lerche, 2015) these methods - while mathematically complex - are available in computationally tractable form (e.g. "Fast-dm-30" of (Voss, Voss \& Lerche, 2015). However, many of these methods require at least 10 trials with each possible outcome; this may require a large number of trials if accuracy is high (so that erroneous responses are rare). A simplified method (the "EZ" model of (Wagenmakers, Van Der Maas \& Grasman, 2007), later extended to the "EZ2" or "robustEZ" model in (Grasman, Wagenmakers \& van der Maas, 2009) uses an approach similar to signal detection theory to estimate DDM parameters using only mean, variance, and accuracy of correct RTs.

DDMs have been used successfully in cognitive neuroscience, to help elucidate differences in cognitive processing between control and patient populations, and to link behavior with neurophysiological data (e.g. functional neuroimaging) to show how brain systems encode and integrate sensory information and use it to generate behavioral responses. An important application of the approach is that such latent cognitive processes may suggest targets for therapy, in patient populations with impaired decision-making. For example, Herzallah et al. (2017) used DDM models to show that patients with Parkinson's disease (PD) have higher response caution a (thus requiring more evidence before making responses), and patients with major depressive disorder (MDD) display reduced drift rate for stimuli associated with positive (put not negative) feedback, while patients with comorbid PD and MDD display both features. The DDM analysis therefore allowed inference of underlying features of the cognitive deficit in these patient groups that were not evident from analyzing observed behavior alone.

We adopted a similar approach here, applying DDM analysis to individual subject data from Experiment 1, and then testing for group differences in extracted parameter values for subjects in the PTSS vs. noPTSS groups. Experiment 1 revealed that the PTSS group performed better than the noPTSS group in the Low spatial separation (most difficult) trials. Most interestingly, this improved performance did not come with an increase in response time, suggesting that the better performance could not simply be attributed to a speed/accuracy tradeoff, with PTSS participants responding more slowly and carefully. In this analysis, our goal was an initial exploration of latent cognitive mechanisms that could produce the observed behavior in which the PTSS group 
453

454

455

456

457

458

459

460

461

462

463

464

465

466

467

468

469

470

471

472

473

474

475

476

477

478

479

480

481

482

483

484

485

486

487

488

489

490

491

492

493

494

outperformed the noPTSS group at the Low spatial separation, but not at the other, easier separations - without a concomitant increase in RT.

\section{METHODS}

Because the behavioral task involved a small number of trials ( 24 per subject), divided among multiple experimental conditions, analysis via "full" DDM was inappropriate, since this requires a minimum of about 10 trials with each outcome (correct and incorrect) for each condition. Instead, we used the "robust-EZ" DDM method (Wagenmakers et al., 2008) which bases analysis on RT for correct trials only and which can be used with a fairly small number of trials. To do this, we grouped trials by spatial separation, resulting in 8 trials per spatial condition. Further, because many subjects performed at or near ceiling on the High (easy) spatial separation, we focused on the Low (difficult) and Medium spatial separation conditions. The robust-EZ DDM method requires calculating mean and variance of reaction time for trials with correct responses (cMRT, cVRT), as well as proportion of trials with incorrect responses (proportion error trials, Pe), so we calculated these summary statistics for each subject based on trials at the Low and Medium spatial separation. Since the robust-EZ method requires Pe $>0$, we were forced to drop data from 16 subjects who had 100\% correct responses at the Medium spatial separation $(n=12)$, the Low spatial separation $(n=1)$, or both $(n=3)$; eight further subjects were excluded who had $<2$ correct responses at the Low $(n=6)$ or Medium $(n=2)$ spatial separation, since RT variance could not be calculated. This left 118 participants for whom all six summary statistics were available. There were no significant differences between these 118 participants and the remaining 25 subjects in gender, group, or PTSS status (chi-square test with Yates correction for $2 \times 2$ tables, all $\chi^{2}<4.0$, all $\left.p>.140\right)$, in age $(t(140)=1.23, p=.223)$ or in task performance including total percent correct, RT on correct trials, or RT on incorrect trials (all $t<2.0$, all $p>0.05$ ).

The six summary variables for each subject were provided to the "robust-EZ" DDM model (Wagenmakers et al., 2008), available as the EZ2 package for R (http://R-Forge.R-project.org). Specific R commands used are provided in the Appendix. The model was used to estimate values of five free parameters for each subject: an overall value of a (boundary separation), $\mathrm{z}$ (starting point), and Ter (nondecision time), as well as separate drift rates v27 and v63 for trials involving low spatial separation $(27 \mathrm{~cm})$ and high spatial separation $(63 \mathrm{~cm})$. These estimated values for each subject are those which provide the best fit to the subject's trial-by-trial data, in terms of minimizing error between the model predictions and the subject's actual behavior (response and RT) across trials.

After obtaining estimated parameter values (v27, v63, z, a, Ter) for each subject, these parameter values were then used as dependent values analyzed via ANOVA, with between-subject factors of gender, group, and PTSS status. For assessing model goodness-of-fit, EZ2 output provides a "value" score for each subject, which is sum of squared prediction error (SSE) multiplied by $10^{\wedge} 7$ for precision; these were converted back to SSE and univariate ANOVA was used to compare goodness of model fit as a function of gender, group, and PTSS status.

\section{RESULTS}

The model converged for all subjects except one (a 28-year-old male civilian; his data were excluded from further analysis. SSE averaged 0.049 (SD 0.037, range 0.004-0.234). There were 
495

496

497

498

499

500

501

502

503

504

505

506

507

508

509

510

511

512

513

514

515

516

517

518

519

520

521

522

523

524

525

526

527

528

529

530

531

532

533

534

535

536

537

538

no differences in goodness-of-fit measure for the model as a function of subject group or PTSS status (univariate ANOVA, all $p>.100$ except group $p=.072$ ). Univariate ANOVAs were conducted on each of the five parameters, with factors of gender, group, and PTSS status. For v27 (drift diffusion rate, Low spatial separation), PTSS participants had higher values than noPTSS subjects $\left(F(1,105)=5.90, p=.017, \eta^{2}=0.053\right)$, with no effects of group or gender and no interactions (all $\mathrm{p}>.100)$. PTSS participants also had higher response caution a $(F(1,105)=$ $4.30, p=.041, \eta^{2}=0.039$ ), with no effects of group or gender and no interactions (all $p$ 's $>.070$ ). There were no effects of group, gender or PTSS status on v63 (drift diffusion rate, Medium spatial separation), starting point $\mathrm{z}$, or nondecision time Ter (all $p>.100$ ). Figure 5 summarizes estimated parameter values, for PTSS and noPTSS groups. Specifically, the PTSS group had higher drift diffusion rate for trials at the Low spatial separation (v27), indicating faster accumulation of evidence, and lower values of response caution (a), indicating less evidence was required before deciding to execute a response. Other parameters including drift diffusion for trials at the Medium spatial separation (v63), starting point (z), and nondecision time (Ter), did not differ between groups.

In summary, experiment 2 used DDM modeling to further examine decision making processes in the spatial separation task given the unexpected result in Experiment 1, where PTSS participants outperformed noPTSS participants in the hardest (Low spatial separation) trials with no slowing of RT. DDM modeling suggested two mechanisms that contributed to performance of the PTSS and noPTSS groups. First, PTSS participants had greater drift diffusion rate on trials with Low spatial separation, indicating faster accumulation of evidence on the more difficult trials (but not on the easier trials at Medium spatial separation). Second, PTSS participants had lower response caution, indicating that less evidence was required before selecting a response. This bias for impulsivity in the PTSS group would be exacerbated by a bias against further accumulation of evidence on the more difficult trials.

\section{GENERAL DISCUSSION}

To determine if poor visual discrimination underlies increased generalization previously observed in Veterans with PTSD we used a visual match-to-sample pattern separation task. Given prior research documenting increased generalization in PTSD, it might be expected that the PTSS group would show correspondingly decreased discrimination, particularly on the more difficult trials. Instead, current results showed that the PTSS group outperformed the noPTSS group on the hardest trials of visual spatial discrimination task, i.e. those trials with the smallest amount of spatial separation.

A simple explanation for this result would have been in terms of a speed/accuracy tradeoff: the PTSS group might have simply increased their accuracy by taking more time to carefully evaluate and respond to stimuli - which would be reflected by slower RT in the PTSS group. However, this was not the case; if anything, the PTSS group responded slightly faster than the noPTSS group. Thus, slower RT cannot explain better discrimination by the PTSS group. To determine which mechanisms might lead to better discrimination without increase in RT, we turned to computational modeling using a DDM model. The DDM model indicated that the PTSS group both accumulated evidence faster (higher drift rate $v$ ) and have lower response caution (reduced boundary separation $a$ between possible responses, indicating reduced response caution). This result indicates that PTSS participants may tend to be impulsive in their decision making and also accumulate evidence faster in selecting a decision; the latter in particular could 
539 promote faster RTs without necessarily entailing a reduction in performance. Future research

540 directly addressing decision-making processes in PTSS is warranted to understand the

541 differential contribution of these processes and their role in PTSS.

542 It is also possible that hippocampal differences previously observed in PTSD are not entirely at

543 odds with the present findings. First, it is important to stress that neuroimaging was not available

544 for participants in the current study; therefore, hippocampal volume reductions could not be

545 verified. A further possibility is that the present task is only relying on a portion of the

546 hippocampal structure. It may be that while the entire hippocampus is smaller, the functions of

547 individual substructures may be altered in different ways. Support for this notion comes from

548 other visual discrimination tasks in healthy participants which have found that pattern separation

549 is more precisely related to the dentate gyrus of the hippocampus (Bakker et al., 2008; Berron et

550 al., 2016). The role of the dentate gyrus in spatial pattern separation is further supported by

551 extensive work using rat models. Gilbert et al. (2001) used a similar spatial delayed-match-to-

552 sample paradigm as we used in our experiment. Using a cheeseboard maze apparatus they

553 required rats to uncover a baited food well (i.e. sample phase), and then following a delay select

554 between one of two wells (i.e. choice phase): one well was the same as the sample phase (correct

555 choice) and a second was varying distances from the sample phase (incorrect choice). Rats with

556 dentate gyrus lesions had impaired performance as the small spatial separations (more difficult

557 trials) but improved as spatial separations increased and the task became easier. Given the

558 similarities between the two tasks, it would be expected that the present task is also reliant

559 specifically on the dentate gyrus, suggesting that differences in dentate gyrus activity may

560 underlie better discrimination performance in the PTSS group. However, given data suggesting

561 that hippocampal volume reduction is a pre-existing risk factor for PTSD (Gilbertson et al.,

562 2002b), rather than emerging in the wake of PTSD or trauma exposure, it is possible that

563 differences in dentate gyrus structural or functional activity may also be a pre-existing risk

564 factor, leading to similar distribution of dentate gyrus impairments among our PTSS and noPTSS

565 participants. Future research using spatial visual discrimination tasks in fMRI to elucidate the

566 contribution of hippocampal substructures in healthy, aging, and PTSD participants is clearly

567 warranted.

568 Another consideration is the type of pattern separation task. Many fMRI studies use pattern

569 separation tasks that ask participants to discriminate highly similar stimuli (Bakker et al., 2008;

570 Lacy et al., 2011; Berron et al., 2016, rather discriminate identical stimuli at different spatial

571 locations (as in the present task). To our knowledge, no study has included both types of task to

572 determine if performance on these tasks is correlated. Further work should examine whether

573 differences observed in discrimination processes in PTSD/PTSS participants in a spatial

574 separation task would carry into other tasks of pattern separation.

575 It is also possible that our pattern separation task was tapping into other cognitive domains that

576 were not necessarily measured or controlled in the present study. Participants in our study were

577 permitted to respond with one or both hands on the keyboard. Therefore, it cannot be ruled out

578 that inhibitory processes or lateralization influences, such as the Simon effect, was playing a role

579 in performance differences. Previous research examining response inhibition in PTSD proposes

580 that a reliance of bottom-up processing and a breakdown of inhibition results in hyperarousal and

581 poor attentional control (Bryant et al., 2005). While our task was one of spatial discrimination, it

582 cannot be denied that it involved a considerable amount of attention and decision-making 
583

584

585

586

587

588

589

590

591

592

593

594

595

596

597

598

599

600

601

602

603

604

605

606

607

608

609

610

611

612

613

614

615

616

617

618

619

620

621

622

623

624

625

processes. Furthermore, poor executive control or response inhibition may also have interacted with participants' handedness preferences. Recent research utilizing a go/no go task measuring inhibitory processes reported that not only were patients with PTSD more likely to make inhibition-related errors than participants without trauma, but the process of inhibition relied on different brain networks such that controls relied on a right-lateralized cortical network while the PTSD participants activated only on the left-lateral frontal cortex during inhibition (Falconer et al., 2008). Additionally, recent research examining the relationship between fear generalization and behavioral pattern separation reports brain areas involved in inhibition and safety processing play a role in behavioral pattern separation including the subcallosal cortex, orbitofrontal cortex, and ventromedial prefrontal cortex (Lange et al., 2017). This is in line with recent work using rat models of PTSD which further implicate the prefrontal cortex as a key region leading to deficits in cognitive flexibility and inhibitory responses (George et al., 2015). Future research more explicitly examining inhibition, decision making, and its relationship to performance in pattern separation tasks in PTSD is necessary.

Finally, there are a number of other factors which may affect cognitive function, such as socioeconomic status and intelligence, which were not controlled for in the current study, but could be examined in future work. Likewise, future studies could examine depressive symptoms and other symptoms which are often comorbid with PTSD, which could be contributing to the current results; in particular, depressed patients have sometimes shown overgeneral memory (e.g. Watkins \& Teasdale, 2001), while a prevalent hypothesis of depression suggests that depression is associated with reduced levels of neurogenesis in the dentate gyrus, potentially affecting pattern separation processes in the dentate gyrus (for review, Samuels \& Hen, 2011). Therefore, it would be very interesting to examine this spatial discrimination task in patients with depression alone or in combination with PTSD symptoms.

Notwithstanding these limitations, our results provided no evidence to suggest that increased generalization in participants with PTSS is due to reduced ability to discriminate. In fact, results indicate the opposite, with PTSS participants showing better discrimination performance. It is important to consider that our expectations for the current study were derived from prior work suggesting that participants with PTSS/PTSD show increased generalization on computer-based associative learning tasks. However, the present task was more precisely a task of discrimination. While generalization and discrimination are usually considered to be (negatively) related, they are not necessarily opponent processes, and as such may depend on different hippocampal substructures (Bakker et al., 2008; Rolls, 2013). Therefore, future research using both generalization and discrimination tasks in the same sample of participants is necessary to determine if the same participants who show heightened generalization are those that also show heightened discrimination.

\section{ACKNOWLEDGEMENTS}

Opinions stated herein do not necessarily represent the official views of the Department of Veterans Affairs or the U. S. Government.

For assistance with subject recruitment and data collection, the authors wish to thank Yasheca Ebanks-Williams. The spatial discrimination task used in the current study was adapted from a version developed by Ray Kesner and colleagues (unpublished), whom the authors wish to thank for helpful discussions during software development and task piloting. An executable version of 
626 the current spatial discrimination task is available as open access software (freely available for 627 non-commercial use) on Open Source Framework (https://osf.io/hpek3/). 
630

631

632

633

634

635

636

637

638

639

640

641

642

643

644

645

646

647

648

649

650

651

652

653

654

655

656

657

658

659

660

661

662

663

664

665

666

667

668

669

670

671

672

673

674

Anastasides N, Beck KD, Pang KCH, Servatius RJ, Gilbertson MW, Orr SP, Myers CE 2015. Increased generalization of learned associations is related to re-experiencing symptoms in Veterans with symptoms of post-traumatic stress. Stress 18:484-489. DOI: $10.3109 / 10253890.2015 .1053450$.

Astur RS, St Germain SA, Tolin D, Ford J, Russell D, Stevens M 2006. Hippocampus Function Predicts Severity of Post-Traumatic Stress Disorder. CyberPsychology \& Behavior 9:234240. DOI: $10.1089 / \mathrm{cpb} .2006 .9 .234$.

Bakker A, Kirwan CB, Miller M, Stark CEL 2008. Pattern separation in the human hippocampal CA3 and dentate gyrus. Science (New York, NY) 319:1640-1642. DOI: 10.1126/science.1152882.

Berron D, Schutze H, Maass A, Cardenas-Blanco A, Kuijf HJ, Kumaran D, Düzel E 2016. Strong Evidence for Pattern Separation in Human Dentate Gyrus. Journal of Neuroscience 36:7569-7579. DOI: 10.1523/JNEUROSCI.0518-16.2016.

Blanchard E, Jones-Alexander J, Buckley TC, Ferneris CA 1996. Psychometric properties of the PTSD checklist (PCL). Behaviour research and therapy 34:669-673. DOI: 10.1016/00057967(96)00033-2.

Blechert J, Michael T, Vriends N, Margraf J, Wilhelm FH 2007. Fear conditioning in posttraumatic stress disorder: evidence for delayed extinction of autonomic, experiential, and behavioural responses. Behaviour research and therapy 45:2019-2033. DOI: 10.1016/j.brat.2007.02.012.

Brewin CR, Holmes EA 2003. Psychological theories of posttraumatic stress disorder. Clinical Psychology Review. DOI: 10.1016/S0272-7358(03)00033-3.

Brewin CR, Gregory JD, Lipton M 2010. Intrusive images in psychological disorders: characteristics, neural mechanisms, and treatment implications. Psychological review. DOI: 10.1037/a0018113.

Bryant, R.A., Felmingham, K.L., Kemp, A.H., Barton, M., Peduto, A.S., Rennie, C., Gordon, E. and Williams, L.M. (2005). Neural networks of information processing in posttraumatic stress disorder: a functional magnetic resonance imaging study. Biological psychiatry, 58(2), 111-118.

Day, H. L., Reed, M. M., \& Stevenson, C. W. 2016. Sex differences in discriminating between cues predicting threat and safety. Neurobiology of learning and memory, 133, 196-203.

Elzinga BM, Bremner JD 2002. Are the neural substrates of memory the final common pathway in posttraumatic stress disorder (PTSD)? Journal of affective disorders. DOI: 10.1016/S0165-0327(01)00351-2.

Falconer, E., Bryant, R., Felmingham, K.L., Kemp, A.H., Gordon, E., Peduto, A., Olivieri, G. and Williams, L.M., 2008. The neural networks of inhibitory control in posttraumatic stress disorder. Journal of psychiatry \& neuroscience: JPN, 33(5), 413.

Fera F, Passamonti L, Herzallah MM, Myers CE, Veltri P, Morganti G, Quattrone A, Gluck MA 2014. Hippocampal BOLD response during category learning predicts subsequent performance on transfer generalization. Human Brain Mapping 35:3122-3131. DOI: $10.1002 / \mathrm{hbm} .22389$.

Gilbert PE, Kesner RP 2002. Role of rodent hippocampus in paired-associate learning involving associations between a stimulus and a spatial location. Behavioral neuroscience.

Gilbert PE, Kesner RP, Lee I 2001. Dissociating hippocampal subregions: A double dissociation between dentate gyrus and CA1. Hippocampus 11:626-636. DOI: 10.1002/hipo.1077. 
Gilbertson MW, Shenton ME, Ciszewski A, Kasai K, Lasko NB, Orr SP, Pitman RK 2002a. Smaller hippocampal volume predicts pathologic vulnerability to psychological trauma. Nature Neuroscience 5:1242-1247. DOI: 10.1038/nn958.

Gilbertson MW, Shenton ME, Ciszewski A, Kasai K, Lasko NB, Orr SP, Pitman RK 2002 b. Smaller hippocampal volume predicts pathologic vulnerability to psychological trauma. Nature Neuroscience 5:1242-1247. DOI: 10.1038/nn958.

Ginsberg JP, Ayers E, Burriss L, Powell DA 2008. Discriminative delay Pavlovian eyeblink conditioning in Veterans with and without posttraumatic stress disorder. Journal of anxiety disorders 22:809-823. DOI: 10.1016/j.janxdis.2007.08.009.

Grasman RPPP, Wagenmakers E-J, van der Maas HLJ 2009. On the mean and variance of response times under the diffusion model with an application to parameter estimation. Journal of Mathematical Psychology 53:55-68. DOI: 10.1016/j.jmp.2009.01.006.

Gurvits TV, Shenton ME, Hokama H, Ohta H, Lasko NB, Gilbertson MW, Orr SP, Kikinis R, Jolesz FA, McCarley RW, Pitman RK 1996. Magnetic resonance imaging study of hippocampal volume in chronic, combat-related posttraumatic stress disorder. Biological psychiatry 40:1091-1099. DOI: 10.1016/S0006-3223(96)00229-6.

Herzallah, M.M., Khdour, H.Y., Taha, A.B., Elmashala, A.M., Mousa, H.N., Taha, M.B., Ghanim, Z., Sehwail, M.M., Misk, A.J., Balsdon, T. and Moustafa, A.A., 2017. Depression Reduces Accuracy While Parkinsonism Slows Response Time for Processing Positive Feedback in Patients with Parkinson's Disease with Comorbid Major Depressive Disorder Tested on a Probabilistic Category-Learning Task. Frontiers in psychiatry, 8.

Holden HM, Hoebel C, Loftis K, Gilbert PE 2012. Spatial pattern separation in cognitively normal young and older adults. Hippocampus 22:1826-1832. DOI: 10.1002/hipo.22017.

Jovanovic, T., Norrholm, S.D., Blanding, N.Q., Phifer, J.E., Weiss, T., Davis, M., Duncan, E., Bradley, B. and Ressler, K., 2010. Fear potentiation is associated with hypothalamicpituitary-adrenal axis function in PTSD. Psychoneuroendocrinology, 35(6), pp.846-857.

Keane, T.M., Fairbank, J.A., Caddell, J.M., Zimering, R.T., Taylor, K.L. and Mora, C.A., 1989. Clinical evaluation of a measure to assess combat exposure. Psychological assessment, 1(1), pp.53-55.

Kostek JA, Beck KD, Gilbertson MW, Orr SP, Pang KCH, Servatius RJ, Myers CE 2014. Acquired equivalence in U.S. Veterans with symptoms of posttraumatic stress: reexperiencing symptoms are associated with greater generalization. Journal of Traumatic Stress 27:717-720. DOI: 10.1002/jts.21974.

Lacy JW, Yassa MA, Stark SM, Muftuler LT, Stark CEL 2011. Distinct pattern separation related transfer functions in human $\mathrm{CA} 3$ /dentate and CA1 revealed using high-resolution fMRI and variable mnemonic similarity. Learning \& Memory 18:15-18. DOI: 10.1101/lm.1971111.

Lange, I., Goossens, L., Michielse, S., Bakker, J., Lissek, S., Papalini, S., Verhagen, S., Leibold, N., Marcelis, M., Wichers, M. and Lieverse, R., 2017. Behavioral pattern separation and its link to the neural mechanisms of fear generalization. Social cognitive and affective neuroscience, 12(11), pp.1720-1729.

Lerche V, Voss A 2016. Model complexity in diffusion modeling: Benefits of making the model more parsimonious. Frontiers in Psychology 7.

Levy-Gigi E, Keri S, Myers CE, Lencovsky Z, Sharvit-Benbaji H, Orr SP, Gilbertson MW, Servatius RJ, Tsao JW, Gluck MA 2012. Individuals with posttraumatic stress disorder show a selective deficit in generalization of associative learning. Neuropsychology 26:758-767. 
DOI: $10.1037 / \mathrm{a} 0029361$.

Levy-Gigi E, Szabo C, Richter-Levin G, Keri S 2015. Reduced hippocampal volume is associated with overgeneralization of negative context in individuals with PTSD. Neuropsychology 29:151-161. DOI: 10.1037/neu0000131.

McLean, C. P., Asnaani, A., Litz, B. T., \& Hofmann, S. G. 2011. Gender differences in anxiety disorders: prevalence, course of illness, comorbidity and burden of illness. Journal of psychiatric research, 45(8), 1027-1035.

Milad, M.R., Pitman, R.K., Ellis, C.B., Gold, A.L., Shin, L.M., Lasko, N.B., Zeidan, M.A., Handwerger, K., Orr, S.P. and Rauch, S.L., 2009. Neurobiological basis of failure to recall extinction memory in posttraumatic stress disorder. Biological psychiatry, 66(12), pp.10751082.

Myers, C.E., Hopkins, R.O., DeLuca, J., Moore, N.B., Wolansky, L.J., Sumner, J.M. and Gluck, M.A., 2008. Learning and generalization deficits in patients with memory impairments due to anterior communicating artery aneurysm rupture or hypoxic brain injury. Neuropsychology, 22(5), p.681.

Myers CE, Kluger A, Golomb J, Ferris S, de Leon MJ, Schnirman G, Gluck MA 2002. Hippocampal atrophy disrupts transfer generalization in nondemented elderly. Journal of geriatric psychiatry and neurology 15:82-90.

Myers CE, Kluger A, Golomb J, Gluck MA, Ferris S 2008b. Learning and generalization tasks predict short-term cognitive outcome in nondemented elderly. Journal of geriatric psychiatry and neurology 21:93-103. DOI: 10.1177/0891988708316858.

Myers CE, Shohamy D, Gluck MA, Grossman S, Kluger A, Ferris S, Golomb J, Schnirman G, Schwartz R 2003. Dissociating hippocampal versus basal ganglia contributions to learning and transfer. Journal of Cognitive Neuroscience 15:185-193. DOI: 10.1162/089892903321208123.

Myers CE, VanMeenen KM, Servatius RJ 2012. Behavioral inhibition and PTSD symptoms in veterans. Psychiatry research 196:271-276. DOI: 10.1016/j.psychres.2011.11.015.

Orr SP, Metzger LJ, Lasko NB, Macklin ML, Peri T, Pitman RK 2000. De novo conditioning in trauma-exposed individuals with and without posttraumatic stress disorder. Journal of Abnormal Psychology 109:290-298. DOI: 10.1037//0021-843X.109.2.290.

Peri T, Ben-Shakhar G, Orr S 2000. Psychophysiologic assessment of aversive conditioning in posttraumatic stress disorder. Biological psychiatry:1-8.

Radell, M.L., Beck, K.D., Gilbertson, M.W. and Myers, C.E., 2017. Post-traumatic stress disorder symptom burden and gender each affect generalization in a reward-and punishmentlearning task. PloS one, 12(2), p.e0172144.

Ratcliff R 1978. A theory of memory retrieval. Psychological review.

Ratcliff R 1993. Methods for dealing with reaction time outliers. Psychological Bulletin 114:510. Ratcliff, R. and McKoon, G., 2008. The diffusion decision model: theory and data for two-choice decision tasks. Neural computation, 20(4), pp.873-922. dx.doi.org 20:873-922. DOI: 10.1162/neco.2008.12-06-420.

Ratcliff, R. and Tuerlinckx, F., 2002. Estimating parameters of the diffusion model: Approaches to dealing with contaminant reaction times and parameter variability. Psychonomic bulletin \& review, 9(3), pp.438-481.

Ratcliff R, Smith PL, Brown SD, McKoon G 2016. Diffusion decision model: current issues and history. Trends in Cognitive Sciences 20:260-281.

Reagh ZM, Yassa MA 2014. Object and spatial mnemonic interference differentially engage 
767 lateral and medial entorhinal cortex in humans. Proceedings of the National Academy of Sciences of the United States of America 111:E4264-E4273.

Rolls, E.T., 2013. The mechanisms for pattern completion and pattern separation in the hippocampus. Frontiers in systems neuroscience, 7.

Samuels, B. A., \& Hen, R. (2011). Neurogenesis and affective disorders. European Journal of Neuroscience, 33(6), 1152-1159.

Shohamy D, Wagner AD 2008. Integrating memories in the human brain: hippocampal-midbrain encoding of overlapping events. Neuron 60:378-389. DOI: 10.1016/j.neuron.2008.09.023. Spreen O, Strauss E 1998. A compendium of neuropsychologial tests. Administration. Tryon WW 1998. A Neural Network Explanation of Posttraumatic Stress Disorder. Journal of anxiety disorders 12:373-385. DOI: 10.1016/S0887-6185(98)00021-8.

van Meurs, B., Wiggert, N., Wicker, I. and Lissek, S., 2014. Maladaptive behavioral consequences of conditioned fear-generalization: a pronounced, yet sparsely studied, feature of anxiety pathology. Behaviour research and therapy, 57, pp.29-37.

Villarreal G, Hamilton DA, Petropoulos H, Driscoll I, Rowland LM, Griego JA, Kodituwakku PW, Hart BL, Escalona R, Brooks WM 2002. Reduced hippocampal volume and total white matter volume in posttraumatic stress disorder. Biological psychiatry 52:119-125.

Voss A, Voss J, Lerche V 2015. Assessing cognitive processes with diffusion model analyses: a tutorial based on fast-dm-30. Frontiers in Psychology 6:470. DOI: 10.3389 /fpsyg.2015.00336.

Wagenmakers E-J, Van Der Maas HL, Grasman RP 2007. An EZ-diffusion model for response time and accuracy. Psychonomic bulletin \& review 14:3-22.

Wagenmakers E-J, Van Der Maas HL, Dolan CV, Grasman RP 2008. EZ does it! Extensions of the EZ-diffusion model. Psychonomic bulletin \& review 15:1229-1235.

Watkins, E., \& Teasdale, J. D. (2001). Rumination and overgeneral memory in depression: Effects of self-focus and analytic thinking. Journal of Abnormal Psychology, 1110, 353-357.

Weathers, F.W., Litz, B.T., Herman, D.S., Huska, J.A. and Keane, T.M., 1993. The ptsd Checklist: Reliability, Validity and Diagnostic Utility. Paper Presented at the Annual Meeting of the International Society for Traumatic Stress Studies. San Antonio, tx.

Werner NS, Meindl T, Engel RR, Rosner R, Riedel M, Reiser M, Fast K 2009. Hippocampal function during associative learning in patients with posttraumatic stress disorder. Journal of psychiatric research 43:309-318. DOI: 10.1016/j.jpsychires.2008.03.011.

Wiecki TV, Sofer I, Frank MJ 2013. HDDM: Hierarchical Bayesian estimation of the driftdiffusion model in Python. Frontiers in neuroinformatics 7. 


\section{Table $\mathbf{1}$ (on next page)}

Demographic information, PTSD scoring, and TMT performance for the veteran and civilian groups.

Note: One civilian participant declined to specify educational level. Asterisk indicates that the non-combat-exposed veteran and civilian groups differed on age (though neither differed from the combat-exposed veteran group. Double asterisk indicates that civilian group differed in PCL score, and rate of PTSS cases, from both veteran groups (which did not differ). 
Table 1. Demographic information, PTSD scoring, and TMT performance for the veteran and civilian groups.

\begin{tabular}{llll}
\hline & $\begin{array}{l}\text { Combat-exposed } \\
\text { veteran }\end{array}$ & $\begin{array}{l}\text { Non-combat- } \\
\text { exposed veteran }\end{array}$ & Civilian \\
\hline $\mathrm{N}$ & 33 & 54 & 55 \\
Gender (\% female) & 3 female (9.1\%) & 13 female (24.1\%) & 40 female (72.7\%) ** \\
Age in years & 49.9 (SD 14.0) & 55.3 (SD 9.9) * & 47.6 (SD 15.4) * \\
Education in years & 15.5 (SD 2.7) & 14.5 (SD 2.2) & 15.1 (SD 3.0) \\
PCL & 56.7 (SD 18.1) & 53.11 (SD 15.9) & $38.2($ SD 14.3) ** \\
PTSS cases & 25 PTSS (75.8\%) & 33 PTSS (61.1\%) & 13 PTSS (23.6\%) ** \\
TMT Part A (sec) & 40.3 (SD 16.4) & 49.8 (SD 22.6) & $48.1(20.3)$ \\
TMT Part B (sec) & 92.9 (SD 38.9) & 110.3 (SD 48.9) & 101.34 (SD 51.5) \\
\hline
\end{tabular}

Note: One civilian participant declined to specify educational level. Asterisks corresponds to independent samples t-tests for continuous values, and $\chi^{2}$ for categorical values. A single asterisk indicates that the non-combat-exposed veteran and civilian groups differed on age (though neither differed from the combat-exposed veteran group. Double asterisk indicates that civilian group differed in distribution of females, PCL score, and rate of PTSS cases, from both veteran groups (which did not differ). 


\section{Figure 1}

Sample screenshots from the discrimination phase of the spatial discrimination task.

A fixation cross appears in the center of the screen for $3 \mathrm{sec}$, followed by a small dot at the target location for that trial. There is a variable delay period, during which the fixation cross re-appears (practice phase 1; not pictured) or else participants complete a digit-naming task, based on numbers that appear for about $1 \mathrm{sec}$ each slightly above or below screen center. Finally, dots appear at target and distractor location, and the participant is asked to use the left or right keys to select the dot which appears at the target location (here, the target dot is on the right).

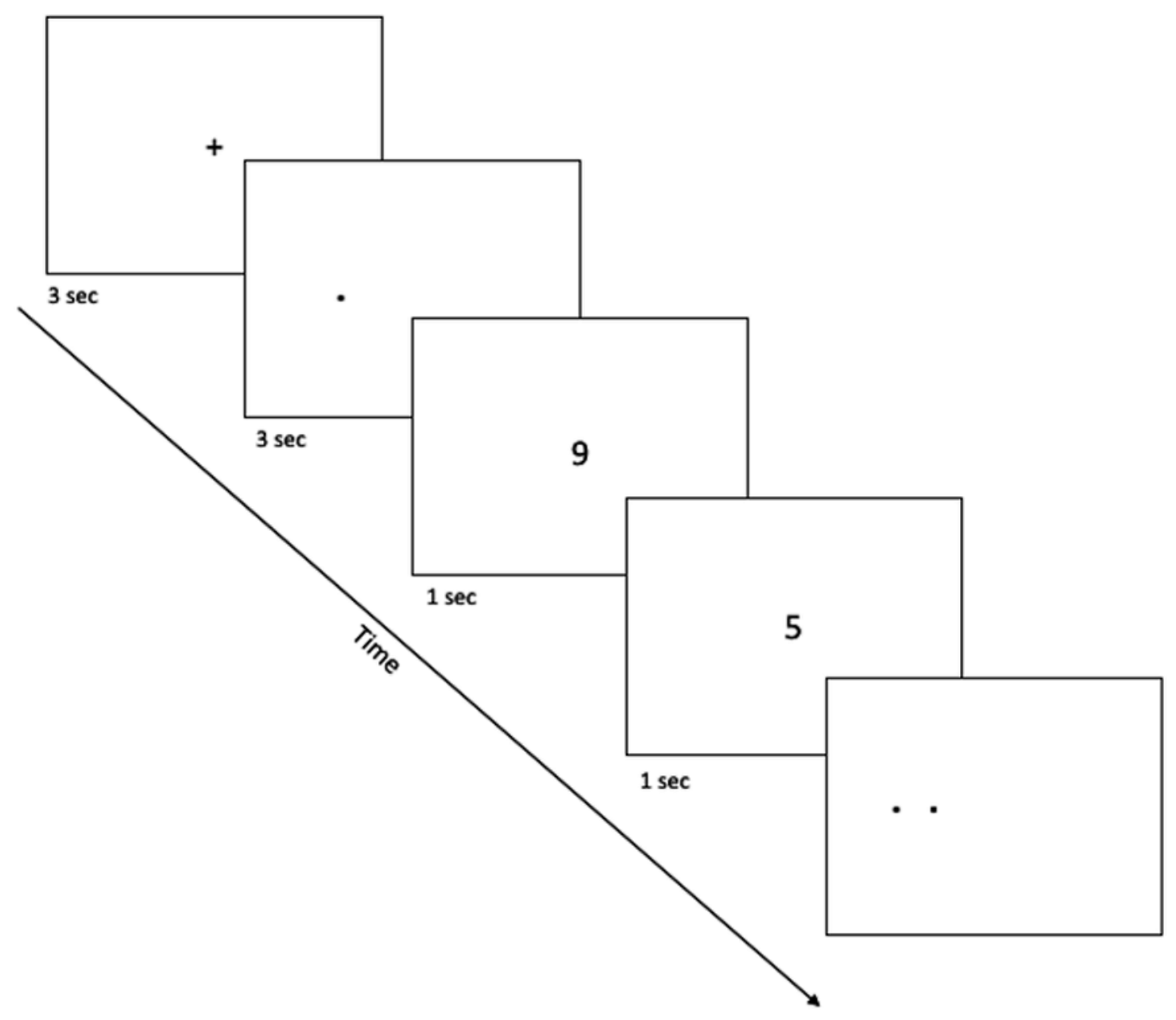


Figure 2

Spatial discrimination task performance of PTSS and noPTSS groups.

(A) On the spatial discrimination task, there was no main effect of temporal delay on response accuracy; however, performance improved as spatial separation between target and distractor increased. (B) Collapsing across temporal delays, participants meeting PTSD symptom criteria (PTSS group) outperformed those not meeting PTSS criteria (noPTSS), specifically at the lowest (i.e. most difficult) spatial separation. Asterisk indicates significant difference between PTSS and noPTSS groups.

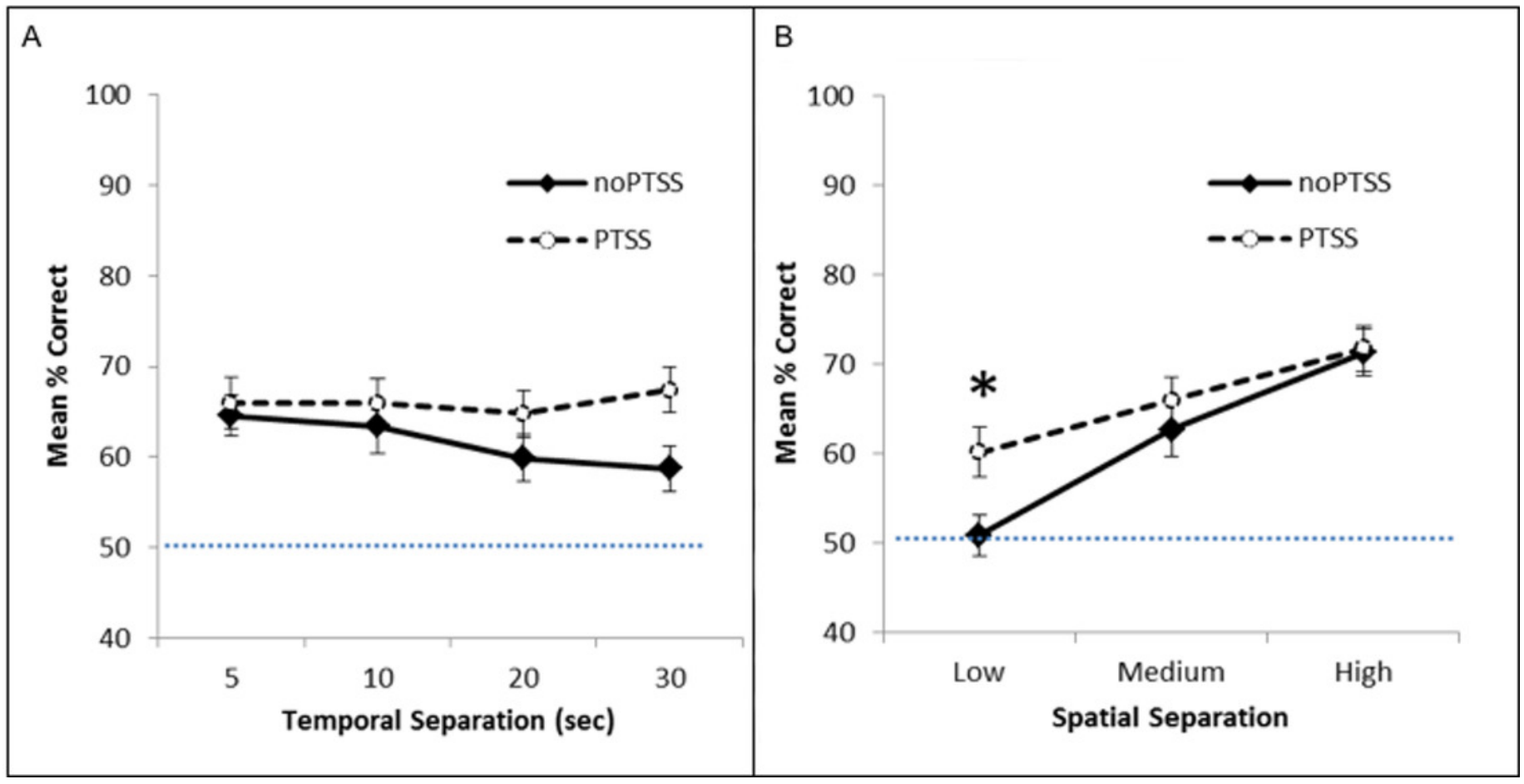




\section{Figure 3}

Response reaction times for the visual discrimination task.

(A) Reaction time (RT) for correct and incorrect responses, on trials at each spatial separation. There was a within-subjects effect of response type (i.e., faster RT for correct than incorrect responses) but no effect of gender or PTSS and no interactions. (B) Analyses were similar when considering mean RT for correct and incorrect trials (regardless of spatial separation), which allowed consideration of all but one subject's data.

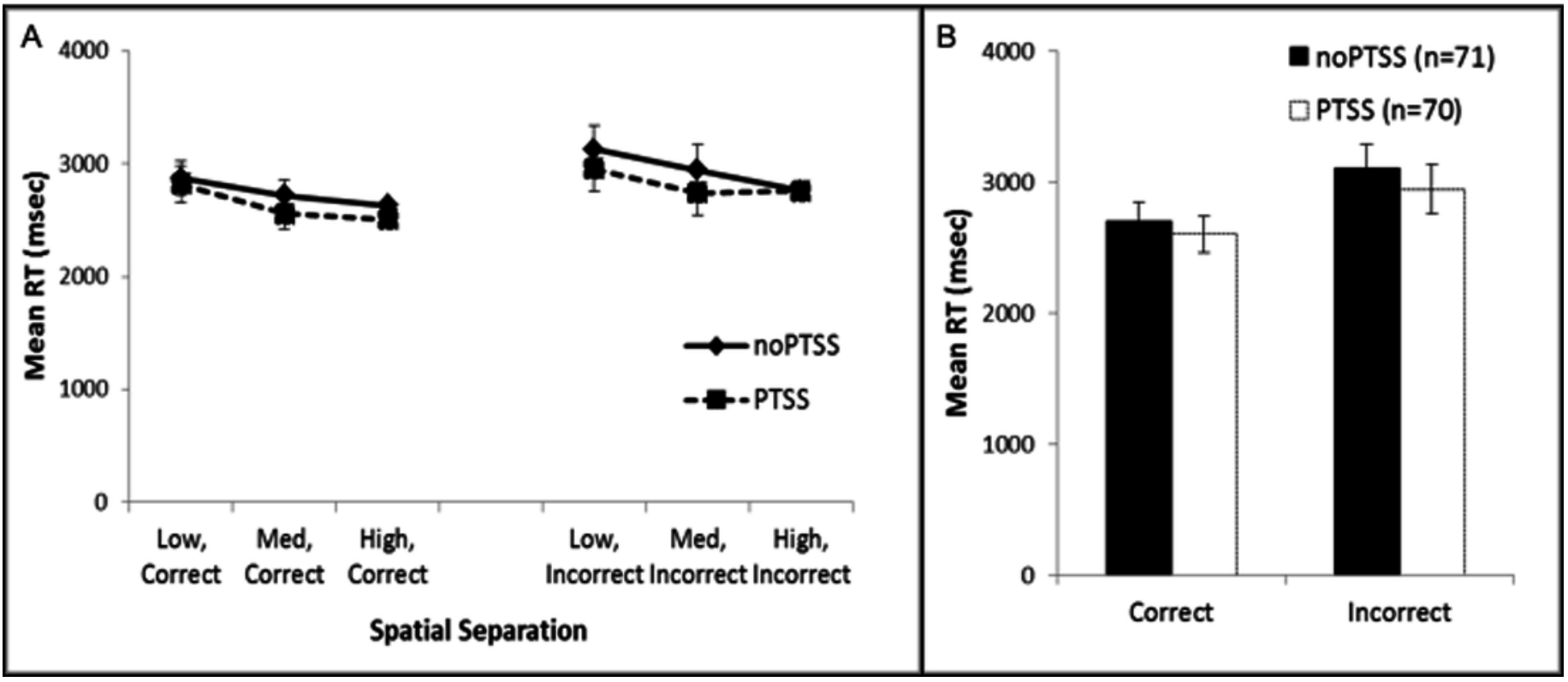




\section{Figure 4}

Schematic of Drift Diffusion Model (DDM) of decision-making.

Each trial starts at time $t_{0}$; noisy information provides evidence which drives the decisionmaking process from a starting point $(z)$ towards boundaries $\theta_{A}$ or $\Theta_{B}$; when a boundary is reached, the corresponding response $A$ or $B$ is selected. The solid blue line schematizes an example trial in which evidence accumulates across time, eventually reaching criterion for response $\mathrm{A}$ at time $\mathrm{t}_{1}$; actual $\mathrm{RT}$ for this trial reflects $\mathrm{t}_{1}$ plus nondecision time Ter (time to encode stimuli and execute motor responses). The green line shows another trial in which stochastic processes lead to a slightly longer decision time $t_{2}$. The red line schematizes a trial in which evidence accumulates favoring response $B$, reaching criterion at time $t_{3}$. Drift rates $V_{A}$ and $V_{B}$ govern the slope of the decision process with higher drift rates producing faster decision times (and faster RTs). Greater boundary separation $\left(a=\theta_{A}-\theta_{B}\right)$ produces "response caution" resulting in greater response accuracy at the expense of longer RTs. Parameters $\mathrm{V}_{\mathrm{A}}$, $\mathrm{V}_{\mathrm{B}}, \mathrm{a}, \mathrm{z}$, and Ter vary across individual subjects but are inferred from observed RT and accuracy data. 
a

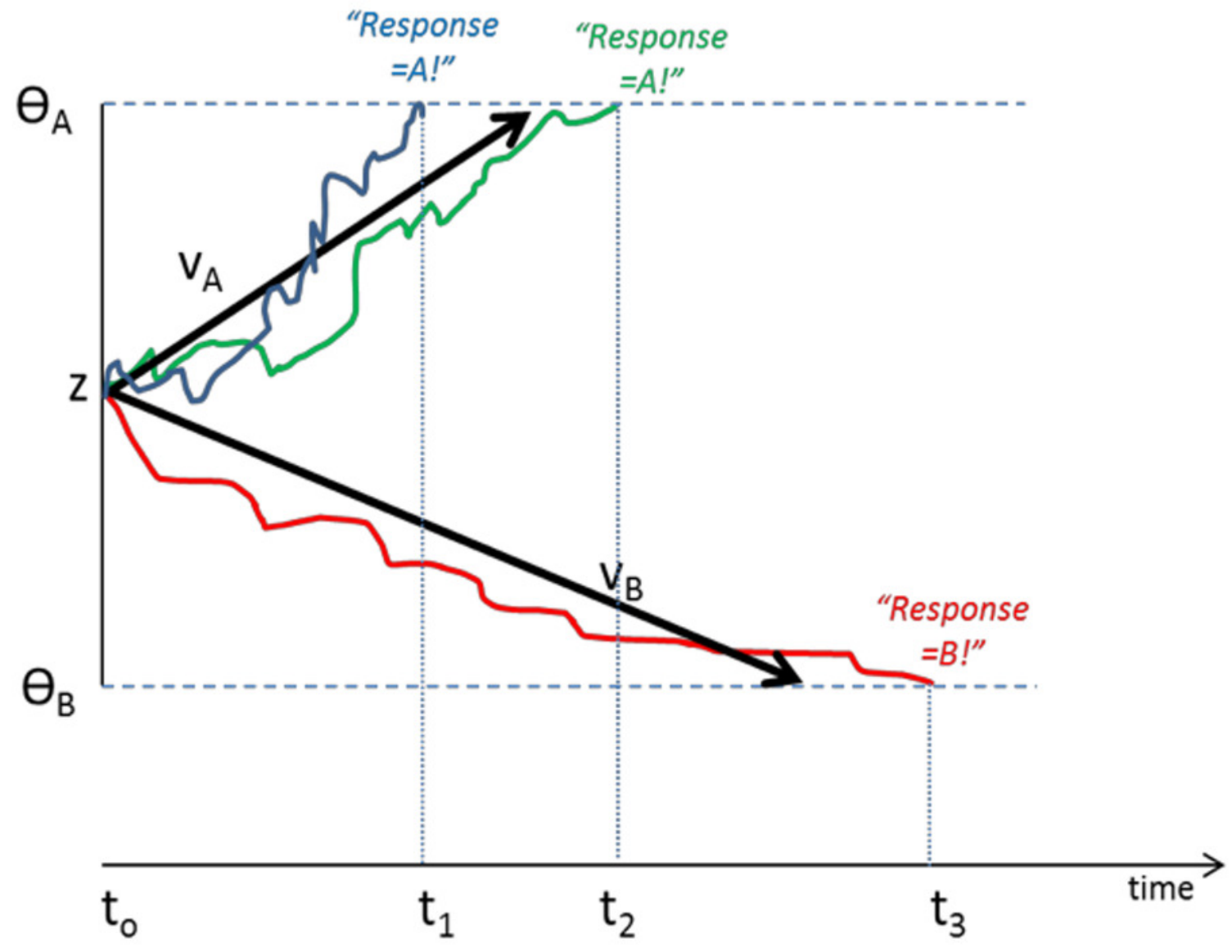




\section{Figure 5}

Estimated parameters, based on drift diffusion model (DDM), for PTSS and noPTSS groups.

There were modest but significant effects of PTSS status on two parameters: v27 (drift diffusion rate at the Low spatial separation) and a (response caution). Specifically, the PTSS group had higher drift diffusion rate for trials at the Low spatial separation (v27), indicating faster accumulation of evidence, and lower values of response caution (a), indicating less evidence was required before deciding to execute a response. Other parameters including drift diffusion for trials at the Medium spatial separation (v63), starting point (z), and nondecision time (Ter), did not differ between groups.

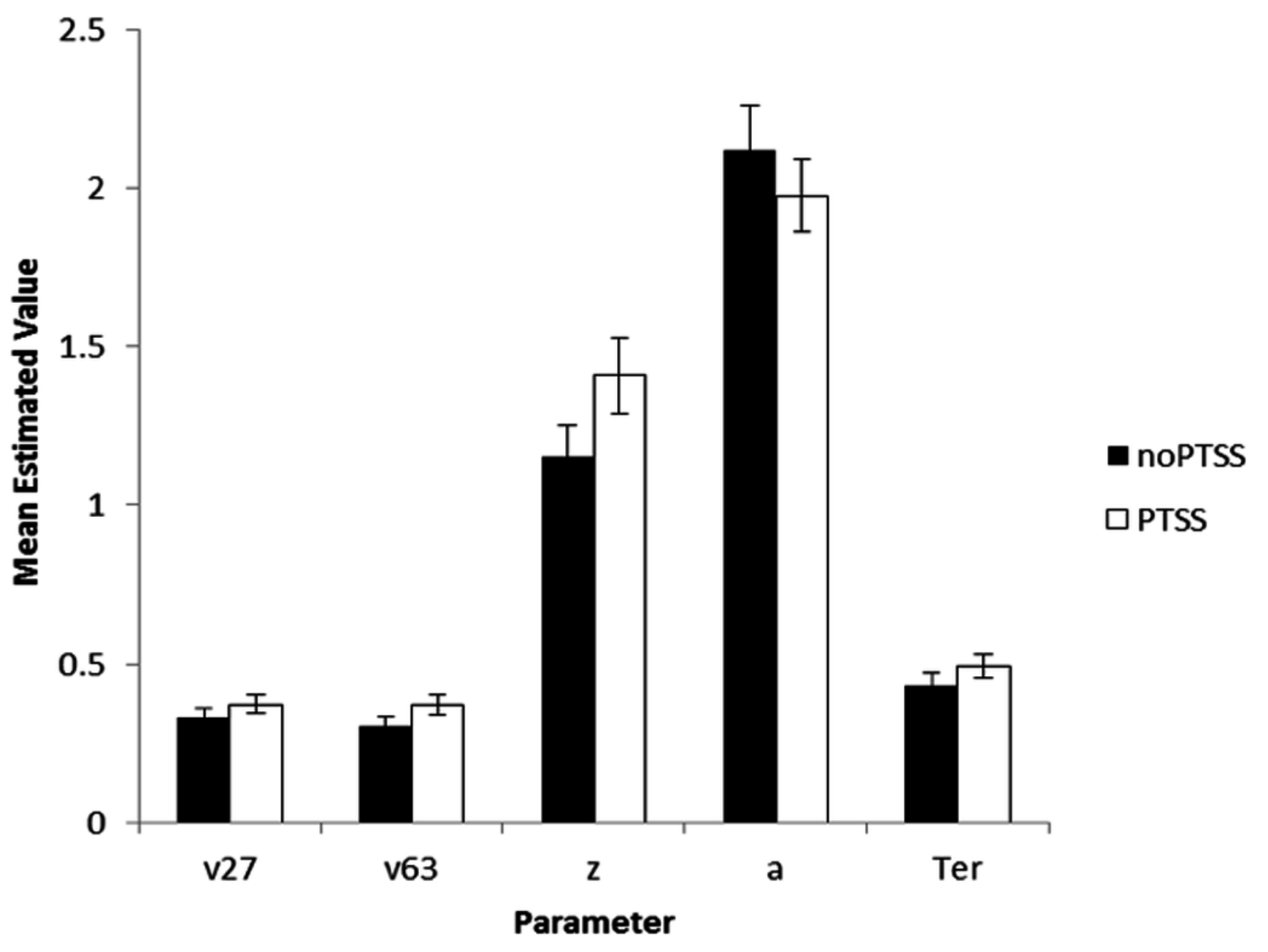

